\title{
Frequency of serotypes of Streptococcus pyogenes in different diseases
}

\author{
E. S. MITCHELL \\ From the Laboratories, Belfast City Hospital, Northern Ireland
}

SYNOPSIS During an 18-month period 1,002 strains of Streptococcus pyogenes, consecutively isolated in the laboratory from a wide variety of lesions, were typed by the precipitin and slideagglutination tests.

The relative frequency of serotypes in different diseases was examined and a close association between type 4 in scarlet fever and type 3.13 in impetigo noted. An apparent lack of any relationship between particular serotypes and streptococcal sore throat was evident.

The difficulty of distinguishing the more virulent types of Str. pyogenes and correlating a typespecific disease relationship is discussed.

Although infections due to Str. pyogenes tend to be less severe now than they were in the first quarter of this century, they still comprise an important group of diseases.

An association between Str. pyogenes and certain diseases, such as scarlet fever, has long been known but as yet there is little general correlation between streptococcal type as identified by the $M$ and $T$ antigens and the clinical picture resulting from infection.

The present investigation was undertaken to determine the frequency of serotypes associated with different manifestations of streptococcal disease.

\section{MATERIAL AND METHODS}

From November 1957 to April 1959, 1,002 strains of Str. pyogenes consecutively isolated in laboratories of the Northern Ireland Hospitals Authority Laboratory Service were submitted for typing. Each laboratory serves at least one general hospital and in many cases also provides a service for examination of specimens received from general practitioners. The sites from which the streptococci were isolated and the diagnoses were established as far as possible at the time of the primary isolation. Second isolates from the same lesion were not included in the investigation.

The types of the strains of Str. pyogenes were identified by the precipitin test (Swift, Wilson, and Lancefield, 1943) and by the slide agglutination technique (Griffith, 1934). Precipitating and agglutinating sera were available for types $1,2,3,5,6,9,12,14,15,17,18,22,23,28$, and Received for publication 19 November 1961.
47. Precipitating but not agglutinating sera were available for types $19,24,26,29,30,43,48$, and 49 , and agglutinating but not precipitating sera for types $4,8,11,13,25,27$, and 44.

\section{RESULTS}

The frequency of the various serotypes in different diseases is set out in the Table. The frequencies that are more than twice or less than half the mean value for the incidence of the type in all infections are indicated by a plus or minus sign provided that the expected number for the cell is 5 or more. The type numbers refer to strains identified by the precipitin or agglutinin tests. Of 1,002 strains typed, 502 $(50.1 \%)$ were identified by the anti-M precipitin test and $484(48.3 \%)$ by slide agglutination only. The remaining 16 strains $(1.6 \%)$ were untypable. Only two strains $(0.2 \%)$ failed to agglutinate and were identified by precipitation.

Among strains isolated from the throat, ears, and air sinuses, the lack of any significant deviation from mean values was very impressive. In impetigo, although the expected number for each cell was less than 5 , the high mean value $(21.4 \%)$ for the nonprecipitating type 3.13 was striking. Types 4 and 6 were noticeably absent. In scarlet fever, types 1 and 12 together accounted for $43.6 \%$ of all strains but neither type showed any significant deviation from mean values. Type 4, however, was significantly in excess whereas types $3.13,6$, and 8 were deficient. There were no representatives of types $2,5,18$, or 28 . 
TABLE

PERCENTAGE FREQUENCY DISTRIBUTION OF TYPES OF STR. PYOGENES IN DIFFERENT DISEASES

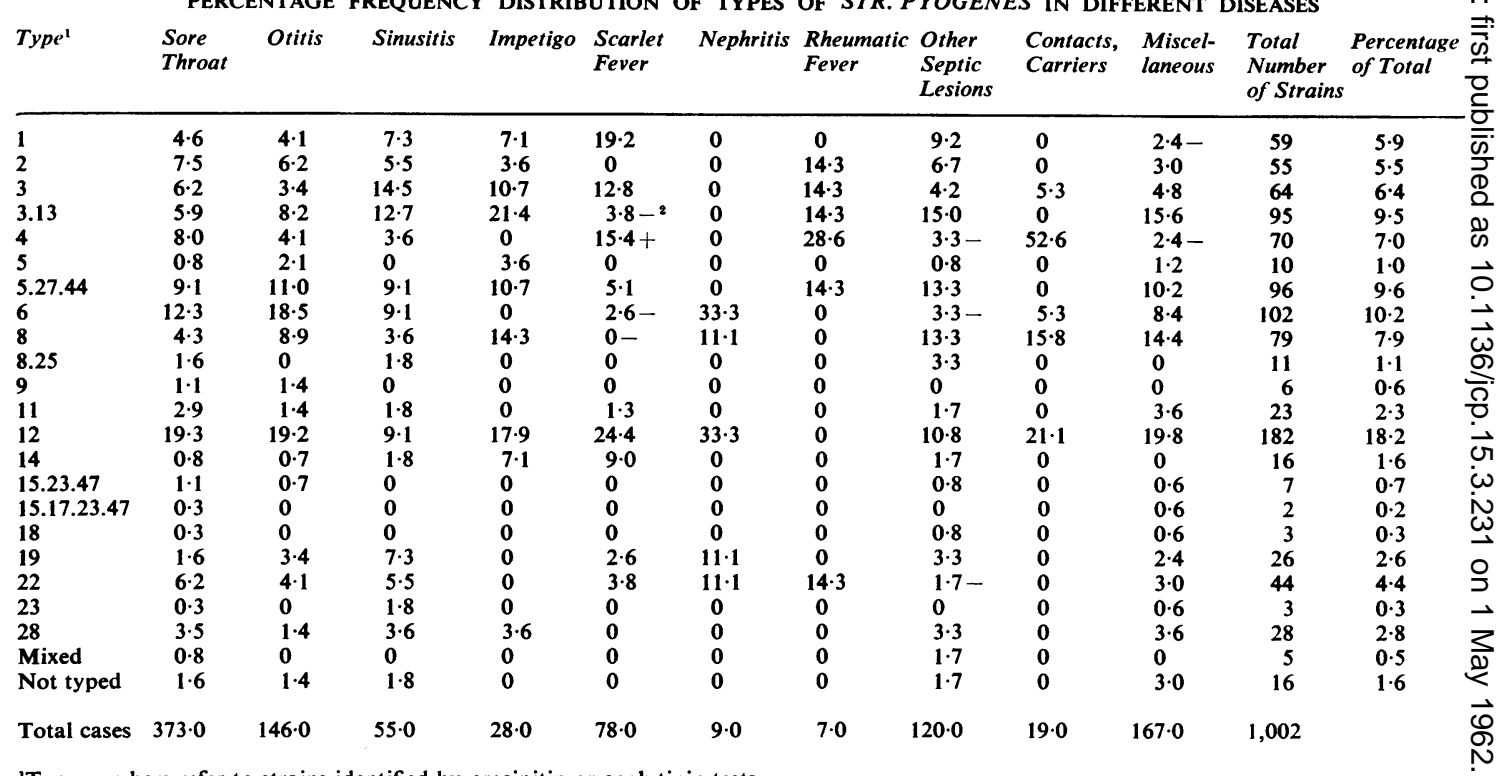

'Type numbers refer to strains identified by precipitin or agglutinin tests.

${ }^{2} \mathrm{~A}$ plus or minus sign denotes more than twice or less than half the mean value (as shown in the last column) entered only where the expected number is 5 or more.

There were nine cases diagnosed as typical acute nephritis. Three of the strains isolated were type 12, three were type 6, and one each of types 19,22 , and 8 , the last strain being identified by agglutination only. All the strains were cultured from throat swabs except one type 6 strain which was recovered from a septic skin lesion.

There were seven cases of rheumatic fever in the series but in two of these the diagnosis was doubtful. Type 4 strains were isolated from two cases, and of the remainder, types $2,3,3.13,5.27 .44$, and 22 accounted for one case each. In all cases the strains were recovered from the throat.

Grouped under other septic lesions, there were 120 strains isolated from abscesses, infected wounds, ulcers, and sinuses from various parts of the body. Types 4,6 , and 22 were significantly deficient in this group.

In the miscellaneous group, there were a variety of conditions including puerperal sepsis, erysipelas, meningitis, lung lesions, etc. Types 1 and 4 were lacking in this group, each accounting for only $2.4 \%$ of all strains (167).

There were four fatal infections attributed to the Str. pyogenes in the series.

\section{ILLUSTRATIVE CASE NOTES}

CASE 1 A thriving boy of $5 \frac{1}{2}$ months died suddenly in a welfare home without any history of previous illness. At necropsy it was established that gastric contents had notâ been aspirated. The lungs on section were grossly oedematous and on culture yielded a profuse growth of Str. pyogenes type 12.

CASE 2 An 11-year-old boy, with no history of previous illness, was found dead in bed. At necropsy, a petechiap rash was present on the chest, but the macroscopie appearance of all organs was normal apart from a con? siderable amount of pulmonary oedema. Cultures were taken from heart blood, lungs, and spleen and in each case Str. pyogenes type 11 was recovered.

CASE 3 A 6-month-old girl was admitted to hospital foro treatment of septic dermatitis. While in hospital the child became pyrexial and died quite suddenly. Stro pyogenes type 12 was isolated from the skin lesions, heart blood, and lungs at necropsy.

CASE 4 A 35-year-old man was admitted to hospital with a diagnosis of frontal sinusitis. Signs of meningismw developed and from the cerebrospinal fluid Str. pyogenes type 5.27.44 was isolated. At necropsy an identical organism was recovered from the heart blood.

\section{DISCUSSION}

The most frequent manifestation of infection with Str. pyogenes is streptococcal sore throat. A striking? feature of 373 such cases observed here was the absence of any significant deviation from mean values? for the various types in all infections. The three types 
most commonly associated with acute sore throat were types 12,6 , and 5.27.44, which accounted for $19 \cdot 3,12 \cdot 3$, and $9 \cdot 1 \%$ respectively of all types causing sore throat. It was, however, also noted that these three types were the most frequently encountered throughout the whole survey representing $18.2 \%$ (type 12), 10.2\% (type 6), and 9.6\% (type 5.27.44) of all strains. All the remaining types were represented in cases of streptococcal sore throat approximately in proportion to the frequency of each type in the whole survey. This would suggest that a simple relationship existed between the most prevalent types of streptococci in the population at the time of sampling, and the number of cases of streptococcal sore throat caused by each type, i.e., the most common types will find susceptible persons and cause sore throat most frequently. It therefore appears that no special relationship exists between any particular serotypes and the development of acute sore throat, but rather given equal opportunity all types behave similarly in this type of streptococcal infection. A lack of any significant deviation from mean values was also observed among strains isolated from the ears and air sinuses so that the distribution of types in these conditions may be dictated in a similar fashion to that governing sore throat.

Hope Simpson (1941) postulated that the causal organism of impetigo contagiosa was an organism special to that disease and suggested the title 'impetigococcus'. Parker, Tomlinson, and Williams (1955), Barrow (1955), and others noted a close association between the type 3.13 streptococcus and impetigo although some of the cases in their series were associated with other streptococcal types.

The 28 cases of impetigo observed in the present investigation comprised sporadic cases from different districts and those affected were mostly children. Type 3.13 was the most frequent and accounted for $21.4 \%$ of all strains, and together with types 12 and 8 comprised over half the impetigo strains isolated. All the type 12 strains yielded $M$ type-specific antigen whereas strains belonging to types 3.13 and 8 could be identified by slide agglutination ( $T$ group antigen) only, so that whatever special relationship exists between these few types and the lesions of impetigo appear to be independent of the M-antigen. While these observations do not entirely support the hypothesis of an 'impetigococcus' it is perhaps true that only a relatively few types of Str. pyogenes are likely to be associated with impetigo.

Schwentker, Janney, and Gordon (1943) have shown, on data collected from various countries, that most of the known serotypes of Str. pyogenes have been associated with cases of scarlet fever. It has, however, been a common experience that out- breaks of this disease are more common with some types than others. The most frequent types isolated from 78 cases observed in this investigation were types $1,3,4$, and 12 , which together accounted for $71.8 \%$ of all scarlatinaform types. Type 4 , however, was the only type which was significantly in excess and this close association was also noted in a survey carried out in England and Wales (Report, 1957). Recurrence of scarlet fever is rare but theoretically possible in view of the occurrence of serologically distinct erythrogenic toxins as shown by Allison and Gunn (1932) and by Coffey (1938). It is thought, however, that the large majority of strains of streptococci produce a toxin with the same antigenic specificity (McCarty, 1954). It seems unlikely, therefore, that the propensity of a few types to cause scarlet fever is due to a differing immune status in the population, so that whether or not the development of a scarlatinaform rash is attributable primarily to the toxigenic capacities of the infecting strains remains to be determined.

Support for the view that a relationship exists between certain serotypes and distinct streptococcal diseases has chiefly been afforded by the close association noted with the group A, type 12 streptococcus and acute glomerulonephritis. Wilmers, Cunliffe, and Williams (1954) have shown that $90 \%$ of 31 cases of acute glomerulonephritis yielded type 12 streptococci on throat culture, whereas types 1,2 , and 3 only accounted for one case each. Other strains which have been incriminated from time to time include types 4,25 , and, more recently, type 49. The last streptococcus, 'red lake', was described by Updyke, Moore, and Conroy (1955).

Of nine cases of acute nephritis observed in this study, only three were associated with the type 12 streptococcus. In an equal number of cases a type 6 strain was cultured. In two cases, from which types 8 and 22 were isolated, the cultures were taken a week after the patients' admission, so that acquisition of the streptococcus in hospital cannot be excluded. It is, however, of interest that the patient harbouring the type 8 streptococcus had suffered two previous attacks of acute glomerulonephritis but the streptococci isolated on those occasions were not typed.

In one case of the present investigation, a type 6 streptococcus was isolated from a pustular skin lesion in a 66-year-old man in whom repeated throat cultures proved negative. At necropsy, acute diffuse glomerulonephritis was establisheu as the cause of death. While most cases of acute glomerulonephritis are preceded by upper respiratory streptococcal infections, it seems possible that the streptococcus in the skin lesions could have sensitized the kidneys.

At present, it appears that infections due to 
certain types of Str. pyogenes, and particularly type 12 , tend to be more often associated with acute nephritis than others. In addition, it seems that different strains of type 12 organisms vary in their capacity to cause nephritis because most upper respiratory type 12 infections are self-limiting diseases and occasionally such infections may be followed by acute nephritis of epidemic proportions, as reported by George, McDonald, Payne, and Slade (1958) and Pleydell and Hall-Turner (1958) in school children.

The implications of these findings are difficult to interpret, but it appears that the development of acute nephritis following upper respiratory streptococcal infections may be due to a factor which is more frequently found in type 12 strains but can on occasion be acquired by a few other types. If this is the case, it might explain why nephritics seldom suffer a recurrence whereas rheumatic patients are susceptible to recurrent attacks of rheumatic fever after infection from practically all types of group A streptococci. This may indicate that in the causation of acute nephritis the peculiarity lies largely in the organisms and that in rheumatic fever it may lie in the host. There is, however, no satisfactory explanation as yet why certain group A strains become capable of causing nephritis or the conditions necessary for sensitization of the kidneys.

Although most infections due to Str. pyogenes are now regarded as mild, four cases of streptococcal septicaemia in this series of 1,002 strains ended fatally. It is interesting to note that all four strains of streptococci associated with these fatal infections belonged to related serotypes of the series $5,11,12$, 27 , and 44 . Only two strains (type 12) were identified by the anti-M precipitin test while types 11 and 5.27.44 were recognized by slide agglutination only. There seems little doubt that the latter two strains were pathogenic yet they failed to produce the $\mathbf{M}$ antigen as indicated by the precipitin test, a fact which casts some doubt on the view that the production of $\mathbf{M}$ antigen is a prerequisite for virulence.
It seems most unlikely that a special relationship exists between the related types $5,11,12,27,44$, and $\%$ a fulminant type of infection, as might be suggested $\vec{F}$ by the four cases observed here, but an extended bacteriological study might well show that fatal infections due to Str. pyogenes are confined to a few⿳亠丷厂 types only.

From these observations it is apparent that if $a^{\stackrel{\Phi}{ }}$ type-specific disease relationship does exist and isco dependent on the $M$ or $T$ antigen, it is only of $a \vec{\circ}$ limited nature. It seems possible, however, that the present failure to correlate particular serotypes with $\vec{\omega}$ the many manifestations of streptococcal diseasemay be due to a streptococcal component, perhaps? as yet unrecognized, which is disease specific ratherc $\vec{c}$ than the $\mathrm{M}$ or $\mathrm{T}$ antigen.

I thank the clinical pathologists of the Northern Ireland Hospitals Authority for sending me strains of streptococciand clinical details of the cases. I also thank Dr. V. D. Allison for initiating the work and for much advice in the preparation of this paper. Supplies of typing sera were kindly supplied by the Streptococcal Reference Labora- $\overrightarrow{0}$ tory, Colindale, London.

\section{REFERENCES}

Allison, V. D., and Gunn, W. (1932). Proc. roy. Soc. Med., 25, 927 Barrow, G. I. (1955). J. Hyg. (Lond.), 53, 495.

Coffey, J. M. (1938). J. Immunol., 35, 121.

George, J. T. A., McDonald, J. C., Payne, D. J. H., and Slade, D. A (1958). Brit. med. J., 2, 1381.

Griffith, F. (1934). J. Hyg. (Camb.), 34, 542.

McCarty, M. (1954). Streptococcal Infections, ed. M. McCarty, p. $130 \Xi$ Columbia Úniv. Press, New York.

Parker, M. T., Tomlinson, A. J. H., and Williams, R. E. O. (1955) J. Hyg. (Lond.), 53, 458.

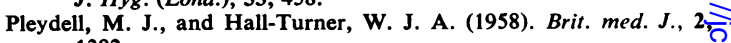
1382.

Report (1957). Monthly Bull. Minist. Hlth Lab. Serv., 16, 163.

Schwentker, F. F., Janney, J. H., and Gordon, J. E. (1943). Amer. J Hyg., 38, 27.

Simpson, R. E. Hope (1941). Lancet, 1, 683.

Swift, H. F., Wilson, A. T., and Lancefield, R. C. (1943). J. exp. Med. $78,127$.

Updyke, E. L., Moore, M. S., and Conroy, E. (1955). Science, 121음 171.

Wilmers, M. J., Cuncliffe, A. C., and Williams, R. E. O. (1954) Lancet, 2, 17. 